Journal of The Korean Astronomical Society

38: $23 \sim 31,2005$

\title{
CN AND CH BAND STRENGTH VARIATIONS IN M71 GIANTS
}

\author{
SANG-GAK LEE \\ Astronomy Program, SEES, Seoul National University, Seoul, Korea 151-742 \\ E-mail:sanggak@astrosp.snu.ac.kr
}

\begin{abstract}
$\mathrm{CN}$ and $\mathrm{CH}$ band strengths for fourteen bright giants in the globular cluster M71 have been measured from archival spectra obtained with the Multiple Mirror Telescope. Adding the collected data from the literature we confirm a bimodality of $\mathrm{CN}$ distribution on the red giant branch and the horizontal branch, and $\mathrm{CN}-\mathrm{CH}$ anti-correlations on the lower giant branch and horizontal branch. However a CN-CH anticorrelation on the upper red giant branch is not quite clear as those of other branches. The small number of statistics could not be excluded as a possible cause. To confirm this, a greater number of sample stars are needed. We also confirm that the ratio of $\mathrm{CN}$-strong to $\mathrm{CN}$-weak stars is quite different from that in 47 Tuc, although the anti-correlation between $\mathrm{CN}$ and $\mathrm{CH}$ bands, the bimodality of the CN distribution, and the spatial distribution of CN stars in M71 are found to be similar to those in 47 Tuc.
\end{abstract}

Key words : globular clusters: individual(M71) — stars: late-type — stars: Population II

\section{INTRODUCTION}

The large scatter of the abundances of $\mathrm{C}, \mathrm{N}, \mathrm{O}, \mathrm{Na}$, $\mathrm{Mg}$, and $\mathrm{Al}$ among globular cluster red giants has tantalized researchers for more than three decades. It has been found that most clusters (except $\omega$ Cen and M22) show negligible internal variations in the abundance of iron group elements, but there are wide variations within the CNO group and among light elements $\mathrm{Na}$, $\mathrm{Mg}$, and $\mathrm{Al}$. The observed abundance variations among cluster giants are far different from those predicted from the classical stellar evolution models and larger than those observed in field giants of similar metallicity. Classical evolution theory (Iben 1964) predicts modest alternations of the surface abundances due to the first dredge-up during the RGB phase. For a solar metallicity star, a factor of two depletion of $\mathrm{C}^{12}$ with a lowering of the $\mathrm{C}^{12} / \mathrm{C}^{13}$ ratio from $90 \sim 100$ to about $20 \sim 30$ and an increase of $\mathrm{N}^{14}$ by a corresponding amount are expected (Iben \& Renzini 1984). In fact, field halo giants of lower RGB stars are found to have abundances of light elements in agreement with predictions from classical evolutionary models. Therefore, the largerthan modest variations among red giants in globular clusters could have originated from either processed materials dredged up to the surface by extra mixing during RGB evolution or primordial and/or accreted and/or polluted inhomogeneous materials.

Although some observational data of evolved red giant stars in the most metal-poor globular clusters M92 (Carbon et al. 1982), M15 (Trefzger et al. 1983), and NGC 6397 (Briley et al. 1990), hint at the deep mixing episode caused by meridional circulation which was proposed early by Sweigart \& Mengel (1979). However, the picture for higher metallicity globular clusters is not so clear. It seems that the processes altering CNO abundance during RGB evolution are sensitive to overall metallicity. The surface abundances of globular cluster RGB stars have revealed a more complex phenomenology. This is likely due to the fact that the surface abundances of these stars are significantly affected by several major factors: deep mixing within individual stars, primordial inhomogeneities within clusters and perhaps accretion and pollution of processed material during the early phases of the cluster evolution.

Some abundance variations found in Turn-Off (TO) and early subgiants, and main-sequence stars in globular clusters are mainly due to the primordial origin and/or accretion and pollution. Especially a $\mathrm{CN}-\mathrm{CH}$ anti-correlation found in main-sequence stars of NGC 6752 (Suntzeff \& Smith 1991), 47 Tuc (Briley 1997; Cannon et al. 1998; Harbeck et al. 2003) and M71 (Cohen 1999; Briley et al. 2001; Briley \& Cohen 2001) and a $\mathrm{O}-\mathrm{Na}$ anti-correlation found in unevolved stars of NGC 6752 (Grattton et al. 2000) and 47 Tuc (Harbeck et al. 2003) could not be explained by an extra deep mixing since main-sequence stars do not have a deep convective envelope down to the burning region. Therefore, if any mixing takes place during red giant branch ascent in M71 it is little and a substantial component of the $\mathrm{C}$ and $\mathrm{N}$ abundance inhomogeneities is in place before the main sequence turn-off (Briley \& Cohen 2001).

M71 is one of the most metal-rich clusters $([\mathrm{Fe} / \mathrm{H}]$ $\sim-0.79$, Sneden et al. 1994) among the Galactic globular clusters. Both CN-strong and $\mathrm{CN}$-weak stars at the same apparent magnitude among M71 giants with luminosities exceeding those of HB stars were discovered by Smith \& Norris (1982). It was found that the CN strengths were anti-correlated with the strengths of the CO near-IR bands, but a positive correlation between $\mathrm{CN}$ and $\mathrm{Na}$ line strengths was shown. Penny et al. (1992) found a bimodal CN-band strength distrib- 
ution and anti-correlated $\mathrm{CN}$ and $\mathrm{CH}$ inhomogeneities among lower RGB stars in M71, and a similar pattern was found by Smith \& Penny(1989) among the red horizontal branch (RHB) stars of this cluster.

Recent spectroscopic study of main sequence stars in M71 (Cohen 1999) showed significant variations in the strength of the $\mathrm{CN}$ band and the $\mathrm{CH}$ band among stars at fixed luminosity in the main sequence of M71. Both $\mathrm{CH}$ and $\mathrm{CN}$ indices appear to be bimodal and they are anti-correlated. These results of M71 are similar to 47 Tuc (Cannon et al. 1998). Both clusters are similar not only in their $\mathrm{CN}$ distribution, but also in that they possess a $\mathrm{CO} / \mathrm{CN}$ anti-correlation, and a positive $\mathrm{Na} / \mathrm{CN}$ correlation. Moreover $\mathrm{C}^{12} / \mathrm{C}^{13}$ ratios of the M71 red giants are found to be low and these ratios are correlated with $\mathrm{CN}$ band strengths such that $\mathrm{CN}$-strong stars have a lower $\mathrm{C}^{12} / \mathrm{C}^{13}$ ratio (Briley et al. 1994, 1997).

The bimodality in the CN band strengths on the giant branch and on the main sequence in 47 Tuc (Norris \& Freeman 1979; Freeman 1985; Paltoglou 1990; Briley 1997; Cannon et al. 1998) has been found from the large sample of stars: the ratio of CN-strong to $\mathrm{CN}$-weak stars is about 2 while the ratio decreases to lower values toward the outer part of the cluster. For M71, Briley et al. (2001) confirmed the bimodal distribution of CN band strengths as DDO C(41-42) color from the sample of 75 red giants down to $\mathrm{Mv}=+2$. The ratio of $\mathrm{CN}$-strong and $\mathrm{CN}$-weak stars on the main sequence is found to be around 1 in M71 (Cohen 1999). Therefore it is of interest to see whether the spatial trends can also be found in M71 and to find the ratio of CN-strong and CN-weak stars for the giant stars in M71. The main object of this study is to add the new data of $\mathrm{CN}$ and $\mathrm{CH}$ band strengths of giants in M71 from archival spectra obtained with the Multiple Mirror Telescope to increase the sample stars with $\mathrm{CN}$ and $\mathrm{CH}$ band strengths in M71. Then we collect the published $\mathrm{CN}$ and $\mathrm{CH}$ band strengths to transform them to one index system to check the anti-correlation between two bands and to find the bimodality distribution of CN band strengths and their spatial distribution. In section 2 measurements of $\mathrm{CN}$ and $\mathrm{CH}$ band strengths from the archival data of the MMT are described; in section 3 the anti-correlation between two bands and the spatial variations of $\mathrm{CN}$ in M71 are presented; in section 4 results are discussed and summarized.

\section{CN AND CH BAND STRENGTH}

We have analyzed archival spectra for fourteen M71 giants to measure the $\mathrm{CN}$ and $\mathrm{CH}$ band strengths. The original spectra were obtained with the Multiple Mirror Telescope for a study of "Distant Halo" (Croswell 1990). The spectra were taken with the blue channel of the MMT spectrograph with an $832 \mathrm{l} / \mathrm{mm}$ grating blazed at $4300 \AA$ to second order. The resolution was $1 \AA$ with 5 pixels per resolution element, and the wavelength coverage ranged from $3600 \AA$ to $4400 \AA$.
The observed spectra were reduced using the standard NOVA package at the CfA (Center for Astrophysics). The wavelength-calibrated spectra were used for this study.

We measured indices $\mathrm{CN}_{(3883)}, \mathrm{CN}_{(4215)}$, and $\mathrm{CH}(\mathrm{G})$ for the sample stars from these spectra. The definitions of the $\mathrm{CN}_{(3883)}, \mathrm{CN}_{(4215)}$, and $\mathrm{CH}(\mathrm{G})$ indices are the same as Lee $(1999,2000)$ 's studies of M3 and M15. These indices are sensitive to the strength of the CN and $\mathrm{CH}$ bands. The $\mathrm{CN}$ indices are the same as those in Smith et al. $(1996,1997)$ and the CH index is almost the same as the CH index in Smith et al. (1996, 1997) except for the continuum part of it. An additional $\langle C N\rangle$ index was defined as an average of the $\mathrm{CN}_{(3883)}$ index and twice the $\mathrm{CN}_{(4215)}$ index in Lee (1999). This implies that in M3 the two indices are strongly correlated in such a way that the $\mathrm{CN}_{(4215)}$ index has a value of one-half the $\mathrm{CN}_{(3883)}$ index.

However in M71 which is a more metal-rich globular cluster than M3, the correlation between $\mathrm{CN}_{(3883)}$ and $\mathrm{CN}_{(4215)}$ indices which is obtained is approximately $\mathrm{CN}_{(3883)}=\sim 2.0 \times \mathrm{CN}_{(4215)}+\sim 0.10$ and the slope is approximately the same as in the case of M3, but overall the $\mathrm{CN}_{(3883)}$ index is shifted to slightly higher than 0.1 . In the derivation of this relation, HB stars, red giants fainter than HB, and I-53 are excluded. I-53 shows either exceptionally strong $\mathrm{CN}_{(4215)}$ compared with other stars of similar $\mathrm{CN}_{(3883)}$ strengths or exceptionally weak $\mathrm{CN}_{(3883)}$ compared with others of similar $\mathrm{CN}_{(4215)}$ strengths. However no proper reason of this is found, leaving open the possibility of a flaw in the original spectrum. So we excluded I-53 for the derivation of the relation between two CN indices.

A similar correlation is found in M15 stars except for the overall shift of the $\mathrm{CN}_{(3883)}$ index by 0.08 to a lower value (Lee 2000). Although the zero point of the $\mathrm{CN}_{(3883)}$ index for a given $\mathrm{CN}_{(4215)}$ index seems to vary in each globular cluster, the overall shift has a small effect on the $\langle C N\rangle$ index (a shift of $\sim$ 0.05 to a higher index value for each star systematically in the case of M71) and does not affect a study of comparisons of $\langle C N\rangle$ indices in a globular cluster. Therefore for the consistency of the index definition, we use the $\langle C N\rangle$ index as defined in Lee (1999). However in the case of the study of several globular clusters of different metallicites, the zero point of the $\mathrm{CN}_{(3883)}$ index in each globular cluster should be taken into account for the comparison between $\mathrm{CN}$ indices of red giants in globular clusters, especially for the case of comparing indices with abundance. The zero point shift seems due to the different abundance effects on $\mathrm{CN}_{(3883)}$ and $\mathrm{CN}_{(4215)}$ indices. As the metallicity of the globular cluster increases, the $\mathrm{CN}_{(3883)}$ index becomes larger for a given $\mathrm{CN}_{(4215)}$ index, while the $\mathrm{CN}_{(4215)}$ index becomes weaker for a given $\mathrm{CN}_{(3883)}$ index. Therefore, even in a globular cluster, if carbon and nitrogen abundances of a star are out of the abundances range of a certain group of stars, the star would not 


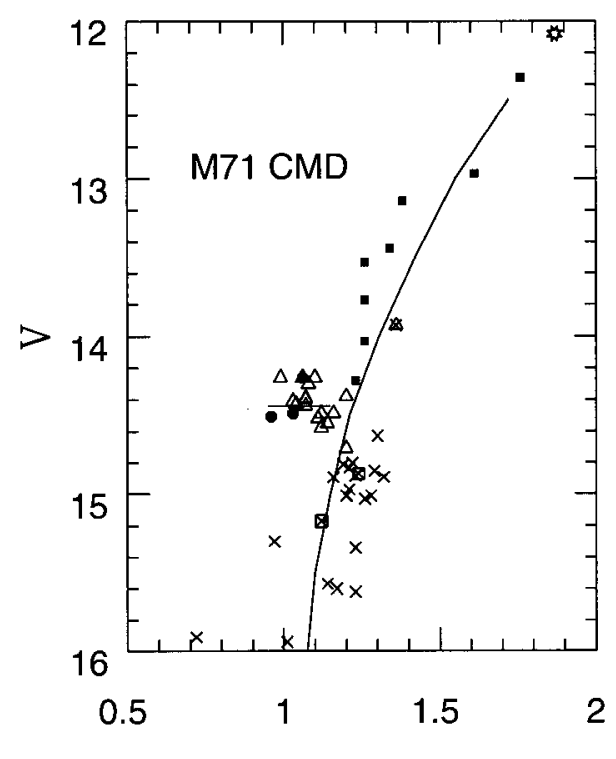

$(B-V)$

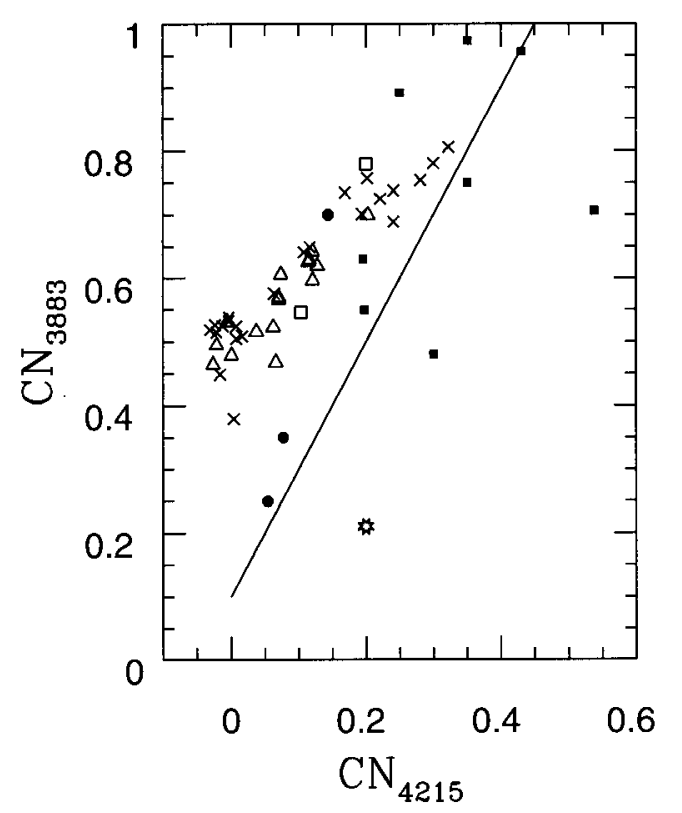

Fig. 2.- Plot of the $\mathrm{CN}_{(3883)}$ index vs. the $\mathrm{CN}_{(4215)}$ index for all stars listed in Table 1. The correlation between two indices for RGB stars shows quite a similar slope to that found in the previous study of M3 (Lee 1999). However there is some amount of shift and also some scatter found for stars collected from the literature, which are HB and lower RGB stars. Symbols used are the same as for Fig.1.

squares, and open squares respectively in Figure 1.

satisfy the correlation between the $\mathrm{CN}_{(3883)}$ index and the $\mathrm{CN}_{(4215)}$ index found for that group of stars. Such stars are found in M15, but not in M71. Nevertheless, we used the same definition of $\langle C N\rangle$, as an average of $\mathrm{CN}_{(3883)}$ and two times $\mathrm{CN}_{(4215)}$ for M71 stars. Each index for original program stars was measured five times and the error of each index is less than 0.01 .

The bright fourteen stars of M71 are listed in Table 1 with collected stars from the literature described in section III. Their designations in column 1 are taken from Arp \& Hartwick (1971) and the star number with prefix KC is from Cudworth (1985). Columns 2 and 3 list the $V$ and $(B-V)$ values of Cudworth (1985). The absolute magnitudes and intrinsic colors are also listed, which were obtained from the previously published values of the distance modulus to M71 and the interstellar reddening toward it. Since the values are quite varied across the literature, we adopted a reddening $\mathrm{E}(B-V)=0.27$ and a distance modulus $(m-M)_{v}$ $=12.80$ (Cudworth 1985). Four indices are listed in the following columns with a final column of remarks.

Among fourteen program stars, I-43, I-54, and I-94 are horizontal branch stars (HB), and the rest of the stars are red giants (RGB) according to their positions in the color-magnitude diagram, but I-109 and KC202 are fainter than $\mathrm{HB}$ and are in about right position of the RGB bump(Cho \& Lee 2002). HB, RGB, and the rest of the stars are represented as filled circles, filled

\section{CN AND CH BAND STRENGTH VARI- ATIONS}

\section{(a) Anti-Correlation of $\mathrm{CN}$ and $\mathrm{CH}$ Band St- rengths}

From literature searches, we could increase the number of stars with $\mathrm{CN}$ and $\mathrm{CH}$ band strengths up to 50 , by transforming indices of other studies to our band index system. Smith \& Penny (1989) and Penny et al. (1992) obtained a set of indices qualifying the strengths of the $\lambda 3883$ and $\lambda 4215 \mathrm{CN}$ bands, the $\lambda 4300 \mathrm{CH}$ band, and the $\mathrm{Ca}$ II $\mathrm{H}$ and $\mathrm{K}$ lines for 16 red horizontal branch stars and for 23 lower giant stars with magnitudes in the range $+0.8<\mathrm{M}_{v}<2.41$ in M71.

We have found one star, I-43 in Smith \& Penny (1989) and two stars, I-109, KC-202 in Penny et al. (1992) overlap with our program stars. Since they both used the same index system, we assumed the indices in the two studies are consistent. So with these three stars, we can transform their indices $\mathrm{S}(3839), \mathrm{S}(4142)$, $\mathrm{m}_{C H}$ to our system indices, $\mathrm{CN}_{(3883)}, \mathrm{CN}_{(4215)}, \mathrm{CH}(\mathrm{G})$, and $\langle C N\rangle$. They are listed in the lower part of Table 1 . Actually the indices for $\mathrm{KC}-22$ were measured in their studies, with differences, -0.055 in $\mathrm{S}(3839)$, 0.007 in $\mathrm{S}\left(4142\right.$ ), and -0.032 in $\mathrm{m}_{C H}$ in the sense of Smith \& Penny's (1989) measurements minus Penny et 
TABLE 1

DATA FOR BRIGHT GAINTS IN M71

\begin{tabular}{|c|c|c|c|c|c|c|c|c|c|}
\hline Star ID & $V$ & $(B-V)$ & $M_{v}$ & $(B-V)_{0}$ & $\mathrm{CN}_{(3883)}$ & $\mathrm{CN}_{(4215)}$ & $<C N>$ & $C H(G)$ & Remark \\
\hline $1-1$ & 14.03 & 1.26 & 0.43 & 0.99 & 0.974 & 0.350 & 0.837 & 0.210 & RGB, ST-CN \\
\hline $1-14$ & 13.77 & 1.26 & 0.17 & 0.99 & 0.630 & 0.196 & 0.511 & 0.280 & RGB, WK-CN \\
\hline $1-43$ & 14.26 & 1.06 & 0.66 & 0.79 & 0.700 & 0.144 & 0.494 & 0.170 & $\mathrm{HB}, \mathrm{ST}-\mathrm{CN}$ \\
\hline $1-44$ & 13.44 & 1.34 & -0.16 & 1.07 & 0.550 & 0.198 & 0.473 & 0.240 & RGB, WK-CN \\
\hline $1-45$ & 12.36 & 1.76 & -1.24 & 1.49 & 0.480 & 0.300 & 0.540 & 0.240 & RGB, WK-CN \\
\hline $1-53$ & 12.97 & 1.61 & -0.63 & 1.34 & 0.706 & 0.538 & 0.891 & 0.280 & $\mathrm{RGB}, \mathrm{ST}-\mathrm{CN}$ \\
\hline $1-54$ & 14.49 & 1.03 & 0.89 & 0.76 & 0.350 & 0.078 & 0.253 & 0.220 & $\mathrm{HB}, \mathrm{WK}-\mathrm{CN}$ \\
\hline $1-56$ & 13.14 & 1.38 & -0.46 & 1.11 & 0.956 & 0.430 & 0.908 & 0.250 & $\mathrm{RGB}, \mathrm{ST}-\mathrm{CN}$ \\
\hline $1-63$ & 13.53 & 1.26 & -0.07 & 0.99 & 0.750 & 0.350 & 0.725 & 0.240 & RGB,ST-CN \\
\hline $1-67$ & 14.28 & 1.23 & 0.68 & 0.96 & 0.892 & 0.250 & 0.696 & 0.240 & $\mathrm{RGB}, \mathrm{ST}-\mathrm{CN}$ \\
\hline $1-94$ & 14.51 & 0.96 & 0.91 & 0.69 & 0.250 & 0.055 & 0.180 & 0.190 & $\mathrm{HB}, \mathrm{WK}-\mathrm{CN}$ \\
\hline $1-109$ & 14.87 & 1.24 & 1.27 & 0.97 & 0.546 & 0.104 & 0.377 & 0.220 & lower RGB, WK-CN \\
\hline $\mathrm{KC}-202$ & 15.17 & 1.12 & 1.57 & 0.85 & 0.780 & 0.200 & 0.590 & 0.190 & lower RGB, ST-CN \\
\hline $\mathrm{V} 2$ & 12.08 & 1.87 & -1.52 & 1.60 & 0.210 & 0.200 & 0.305 & 0.250 & variable, WK-CN \\
\hline $1-3$ & 14.38 & $1: 20$ & 0.78 & 0.93 & 0.570 & 0.071 & 0.356 & 0.209 & RGB, WK-CN \\
\hline $1-11$ & 14.81 & 1.19 & 1.21 & 0.92 & 0.533 & -0.003 & 0.264 & 0.213 & lower RGB, WK-CN \\
\hline $1-28$ & 14.97 & 1.21 & 1.37 & 0.94 & 0.449 & -0.016 & 0.209 & 0.208 & lower RGB, WK-CN \\
\hline $1-48$ & 14.39 & 1.07 & 0.79 & 0.80 & 0.606 & 0.074 & 0.377 & 0.189 & $\mathrm{HB}, \mathrm{ST}-\mathrm{CN}$ \\
\hline $1-55$ & 14.26 & 1.10 & 0.66 & 0.83 & 0.524 & -0.116 & 0.246 & 0.205 & HB, WK-CN \\
\hline $1-58$ & 14.88 & 1.23 & 1.28 & 0.96 & 0.738 & 0.241 & 0.610 & 0.202 & lower RGB, ST-CN \\
\hline $1-59$ & 14.63 & 1.30 & 1.03 & 1.03 & 0.576 & 0.064 & 0.352 & 0.217 & lower RGB, WK-CN \\
\hline $1-61$ & 14.58 & 1.12 & 0.98 & 0.85 & 0.495 & -0.021 & 0.227 & 0.202 & HB, WK-CN \\
\hline $1-62$ & 14.49 & 1.12 & 0.89 & 0.85 & 0.523 & 0.063 & 0.325 & 0.203 & $\mathrm{HB}, \mathrm{WK}-\mathrm{CN}$ \\
\hline $1-75$ & 14.85 & 1.29 & 1.25 & 1.02 & 0.781 & 0.300 & 0.691 & 0.198 & lower RGB, ST-CN \\
\hline $1-88$ & 14.26 & 0.99 & 0.66 & 0.72 & 0.597 & 0.121 & 0.420 & 0.177 & HB, ST-CN \\
\hline $1-111$ & 14.89 & 1.32 & 1.29 & 1.05 & 0.689 & 0.241 & 0.586 & 0.222 & lower RGB, ST-CN \\
\hline $\mathrm{KC}-22$ & 13.93 & 1.36 & 0.33 & 1.10 & 0.625 & 0.115 & 0.428 & 0.213 & RGB, WK-CN \\
\hline $\mathrm{KC}-118$ & 14.55 & 1.14 & 0.95 & 0.87 & 0.479 & 0.001 & 0.241 & 0.197 & $\mathrm{HB}, \mathrm{WK}-\mathrm{CN}$ \\
\hline KC-119 & 14.52 & 1.11 & 0.92 & 0.84 & 0.531 & -0.005 & 0.261 & 0.200 & $\mathrm{HB}, \mathrm{WK}-\mathrm{CN}$ \\
\hline $\mathrm{KC}-126$ & 14.71 & 1.20 & 1.19 & 0.93 & 0.566 & 0.071 & 0.354 & 0.209 & lower RGB, WK-CN \\
\hline $\mathrm{KC}-127$ & 14.44 & 1.07 & 0.84 & 0.80 & 0.630 & 0.116 & 0.431 & 0.178 & $\mathrm{HB}, \mathrm{ST}-\mathrm{CN}$ \\
\hline $\mathrm{KC}-130$ & 15.01 & 1.28 & 1.41 & 1.01 & 0.509 & 0.017 & 0.272 & 0.211 & lower RGB, WK-CN \\
\hline $\mathrm{KC}-136$ & 14.80 & 1.22 & 1.20 & 0.95 & 0.806 & 0.322 & 0.725 & 0.197 & lower RGB, ST-CN \\
\hline KC- 137 & 15.91 & 0.72 & 2.31 & 0.45 & 0.379 & 0.004 & 0.194 & 0.170 & WK-CN \\
\hline $\mathrm{KC}-141$ & 15.34 & 1.23 & 1.74 & 0.96 & 0.505 & 0.008 & 0.261 & 0.211 & lower RGB, WK-CN \\
\hline $\mathrm{KC}-152$ & 15.03 & 1.26 & 1.43 & 0.99 & 0.527 & -0.023 & 0.241 & 0.221 & lower RGB, WK-CN \\
\hline $\mathrm{KC}-155$ & 14.89 & 1.16 & 1.29 & 0.89 & 0.754 & 0.281 & 0.658 & 0.198 & lower RGB, ST-CN \\
\hline $\mathrm{KC}-169$ & 14.30 & 1.08 & 0.70 & 0.81 & 0.699 & 0.203 & 0.553 & 0.178 & $\mathrm{HB}, \mathrm{ST}-\mathrm{CN}$ \\
\hline $\mathrm{KC}-170$ & 15.57 & 1.14 & 1.97 & 0.87 & 0.515 & -0.021 & 0.237 & 0.209 & lower RGB, WK-CN \\
\hline $\mathrm{KC}-183$ & 15.57 & 1.14 & 1.97 & 0.87 & 0.725 & 0.221 & 0.584 & 0.193 & lower RGB, ST-CN \\
\hline $\mathrm{KC}-191$ & 15.01 & 1.20 & 1.41 & 0.93 & 0.525 & -0.012 & 0.251 & 0.216 & lower RGB, WK-CN \\
\hline $\mathrm{KC}-196$ & 15.60 & 1.17 & 2.00 & 0.90 & 0.701 & 0.194 & 0.545 & 0.199 & lower RGB, ST-CN \\
\hline $\mathrm{KC}-215$ & 15.30 & 0.97 & 1.70 & 0.70 & 0.519 & -0.030 & 0.230 & 0.205 & lower RGB, WK-CN \\
\hline $\mathrm{KC}-302$ & 15.62 & 1.23 & 2.02 & 0.96 & 0.525 & 0.008 & 0.271 & 0.213 & lower RGB, WK-CN \\
\hline A3 & 14.43 & 1.04 & 0.83 & 0.77 & 0.468 & 0.067 & 0.301 & 0.207 & $\mathrm{HB}, \mathrm{WK}-\mathrm{CN}$ \\
\hline $\mathrm{C}$ & 14.49 & 1.16 & 0.89 & 0.89 & 0.516 & 0.038 & 0.296 & 0.214 & HB, WK-CN \\
\hline $\mathrm{D}$ & 15.94 & 1.01 & 2.34 & 0.74 & 0.735 & 0.169 & 0.537 & 0.197 & lower RGB, ST-CN \\
\hline K & 14.83 & 1.21 & 1.23 & 0.94 & 0.538 & -0.003 & 0.239 & 0.213 & lower RGB, WK-CN \\
\hline $\mathrm{L}$ & 14.41 & 1.03 & 0.81 & 0.76 & 0.465 & -0.026 & 0.207 & 0.197 & $\mathrm{HB}, \mathrm{WK}-\mathrm{CN}$ \\
\hline $\mathrm{X}$ & 14.40 & 1.07 & 0.80 & 0.80 & 0.642 & 0.121 & 0.442 & 0.187 & $\mathrm{HB}, \mathrm{ST}-\mathrm{CN}$ \\
\hline
\end{tabular}


al.'s (1992) measurements. Therefore we expect those amount of uncertainty for their indices. We use the average values for $\mathrm{KC}-22$.

Figure 1 is the color-magnitude diagram of M71 with the giant branch fiducial line taken from Table 3 of Hodder et al. (1992). The original program stars are represented as filled circles, filled squares, and open squares for HB stars, bright RGB stars, and RGB stars fainter than $\mathrm{HB}$ respectively. $\mathrm{V} 2$ is represented as a starred mark. Stars from Smith \& Penny (1989), mostly $\mathrm{HB}$ stars, are represented as open triangles, while those of Penny et al. (1992), all fainter than $\mathrm{HB}$, as crosses.

Although the plot of $\mathrm{CN}_{(3883)}$ versus $\mathrm{CN}_{(4215)}$ for 50 sample stars in Figure 2 shows the zero points and the slope of the relation between two indices are slightly different from those obtained only from bright giants of original program stars, we took the $\langle C N\rangle$ index as an average of $\mathrm{CN}_{(3883)}$ index and two times the $\mathrm{CN}_{(4215)}$ index as in the previous section for consistency. Since the stars used for index transformation are not all bright giants, it is difficult to correct the different effects of temperature and luminosity on both bands. Since we intend to estimate the deviation from the average strength of $\mathrm{CN}$ for a given temperature and luminosity, the $\langle C N\rangle$ index used in this study would not affect the results of the following analysis.

The $\langle C N\rangle$ versus $M_{v}$ and $(B-V)$ behaviors are plotted in Figures $3-\mathrm{a}$ and $3-\mathrm{b}$, respectively, and the corresponding results for the $\mathrm{CH}(\mathrm{G})$ indices in Figures 3 -c and 3-d. The same symbols are used in the figures as in Figure 1.

There are luminosity dependencies and strong hints of bimodality of CN strength in Figure 3-a with the separation between the "normal" and "enhanced" CN stars as shown in the $[\mathrm{C}(41-42), V]$ diagram in Figure 11 of Smith \& Norris (1982), and also a strong color dependence is found in Figure 3 -b if we ignore the reddest two stars. The $\mathrm{CH}(\mathrm{G})$ index also shows some dependence on luminosity and color as is seen in Figures $3-c$ and $3-\mathrm{d}$.

A plot of the the $\langle C N\rangle$ index against the $\mathrm{CH}(\mathrm{G})$ index in Figure 4-a shows two parallel trends. This figure is similar to Figures 3 and 4 of Cannon et al. (1998) for 47 Tuc stars. As they did, we divide stars empirically into two classes using Fig. 4-b. The stars lower right parts are labelled CN-strong and the upper left CN-weak. Cannon et al. (1998) showed that decreasing the carbon or increasing the nitrogen abundance moves points towards the lower right-hand corner, and vice versa for synthetic spectra. The results of the classification of the sample stars are listed in the last remark column of Table 1 and are illustrated in Figure 4-b with filled circles representing the CN-strong stars and open circles the CN-weak stars. The empirical line dividing the two classes is also plotted in Figure 4-a.

In the above method, I-45 is grouped with the CNweak stars. However it was classified as a CN-strong star in Briley et al. (1994, 1997). Recently Ramirez \& Cohen (2002) deduced high $\mathrm{C}$ abundances for I-45 and I-66 from the analysis of spectra of high dispersion $(\mathrm{R}=35,000)$, obtained with HIRES at the Keck Observatory with suspecting their results due to the fact that the CI lines used for their analysis, may be blended with or completely dominated by lines from the red system of CN in stars with $T_{\text {eff }}<\sim 4200 \mathrm{~K}$. However Briley et al. (1994) found that good fits to the observed spectra of I-45 can be obtained with the O abundances from Sneden et al. (1994) and a larger N abundance for arbitrarily low values of $[\mathrm{C} / \mathrm{Fe}]=-0.6$. $\mathrm{I}-45$ has the lowest value of $[\mathrm{O} / \mathrm{Fe}]=+0.13$ and has the second highest value of $[\mathrm{Na} / \mathrm{Fe}]=+0.49$ among the stars, in the abundance study of M71 giants by Sneden et al. (1994). However we found I-45 is well suited as a $\mathrm{CN}$-weak star in the following $\langle C N\rangle$ index versus magnitude diagram, but not well suited in the $\mathrm{CH}(\mathrm{G})$ versus magnitude diagram. That means our $\mathrm{CH}(\mathrm{G})$ index is not as large as that expected for a $\mathrm{CN}$-weak star if it follows the CN-CH anti-correlation. Therefore if the high $\mathrm{C}$ abundance result of Ramirez \& Cohen (2002) is valid, the large $N$ abundance of Briley et al. (1994) and our low value of $\mathrm{CH}(\mathrm{G})$ index for $\mathrm{I}-45$ need to be checked. If our low $\mathrm{CH}(\mathrm{G})$ index is due to its low temperature, and the large $\mathrm{N}$ abundance of Briley et al. (1994) is due to adopting arbitrarily low values of the $\mathrm{C}$ abundance, we could not exclude the possibility that $\mathrm{I}-45$ is a low $\mathrm{N}$ and high $\mathrm{C}$ abundance star of low temperature, although it requires a more detailed high resolution spectroscopic study for confirmation of it's $\mathrm{N}$ and $\mathrm{C}$ abundances.

Since these two indices, $\langle C N\rangle$ and $\mathrm{CH}(\mathrm{G})$ depend on temperature and luminosity as well as on the relevant abundances, we plot the index-magnitude diagrams for the M71 stars in Figures 5-a and 5-b. In each panel, the same symbols are used as in Fig. 4-b to distingush between the CN-strong and CN-weak stars. These panels resemble conventional CMDs, only with index replacing color as abscissa, showing that both indices are dependent on both temperature and luminosity. Especially, the CN index versus Mv diagram of Fig. 5-a does show a clear dichotomy with surprisingly clear and separate $\mathrm{HB}$ and RGB for each class. Although this result is somehow expected since the primary selection criterion for defining the two classes is the $\langle C N\rangle$ index, it strongly confirms that two classes exist in the lower RGB all the way to the bright RGB as well as in $\mathrm{HB}$ with an approximately equal amount of difference in the $\langle C N\rangle$ index along the RGB and a somewhat smaller amount of it in HB.

The amount of the $\langle C N\rangle$ difference between the two classes is 0.357 for RGB stars with a dispersion of 0.043 for weak-CN RGB stars and 0.042 for strong-CN RGB stars, and 0.183 for HB stars with a dispersion of 0.053 for weak-CN HB stars and 0.056 for strong-CN HB stars. In this analysis, we exclude a variable star, $\mathrm{V} 2$, and $\mathrm{KC}-137$. V2 is not only variable but also the brightest and reddest star among the M71 stars, so it's 

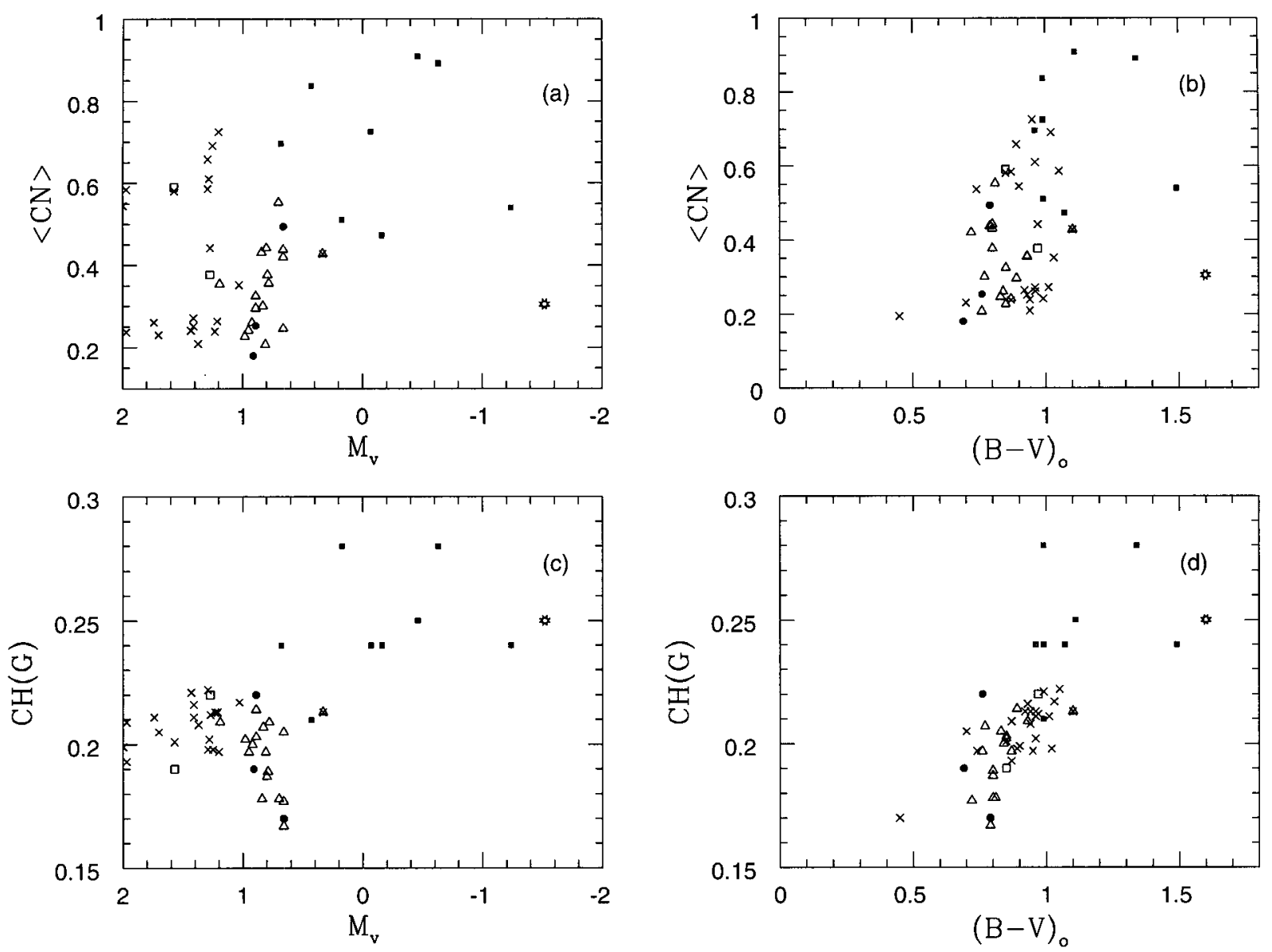

Fig. 3.- $<C N>$ and $\mathrm{CH}(\mathrm{G})$ indices are plotted as a function of absolute magnitude and (B-V) color for all stars listed in Table 1. Symbols used are the same as for Fig. 1.

$\mathrm{CN}$ and $\mathrm{CH}$ band strengths may be affected more by extremely low temperature and gravity. On the other hand, KC-137 has color $(B-V)_{o}=0.45$ and an absolute magnitude $M_{V}=2.31$, which is too blue to be a RGB star and too faint to be a HB star. In addition to that, the membership probability of $67 \%$ (Cudworth 1985 ) is lower than any other sample star. Even if KC137 is a member of M71, it is too blue to have accurate measurements of CN and $\mathrm{CH}$ band strengths. So we exclude these two stars in estimating the index difference between two groups. However the varying differences between two groups for RGB stars and HB stars do not directly imply the differences in nitrogen abundance between two classes in the HB stars are smaller than in RGB stars since $\mathrm{CN}$ band strengths are related in a complicated way with the CNO abundances as well as with the temperature and luminosity. Elucidation requires detailed synthetic spectrum analysis with stellar model atmospheres.

In the corresponding plot for the $\mathrm{CH}$ index of Fig. 5$\mathrm{b}$, we also found clear and separate branches, in the HB and the lower RGB for the weak-CN stars and strong$\mathrm{CN}$ stars but with a somewhat unclear separation in the brighter part of the RGB. In comparison with Fig.5-a we confirm a strong anti-correlation between the two indices in $\mathrm{HB}$ and the lower RGB with one exception star, I-111, but the trend appears not to be confirmed in the upper RGB. The average $\mathrm{CH}(\mathrm{G})$ index for weakCN stars in HB is 0.204 with a dispersion of 0.008 , while that for strong-CN stars is 0.180 with a dispersion of 0.006 . Therefore the difference between the average indices of the two groups in HB is 0.024 , which is more than 3 sigmas for each group. For the fainter part of the RGB, the average index for weak-CN stars is 0.212 with a dispersion of 0.004 and that for strong-CN stars (excluding I-111) is 0.197 with a dispersion of 0.003 . We also found a difference of 0.015 , which is also more than 3 sigmas for each group. Star I-111 is a strong-CN star, but has a strong $\mathrm{CH}(\mathrm{G})$ index too. However the original indices for the $\mathrm{CN}$ and $\mathrm{CH}$ band are collected from Table 1 of Penney et al. (1992). Their values of $\mathrm{S}(3839)$ and $\mathrm{S}(4142)$ for $\mathrm{CN}$ bands as well as values of $\delta \mathrm{S}(3839)$ and $\delta \mathrm{S}(4142)$ indicate it is a CN-strong star, which is also seen in their Figure 4. However the value of $m_{C H}$ for the CH band was listed as 0.290 in their Table 1 , which value is very large for a $\mathrm{CN}$-strong star. 

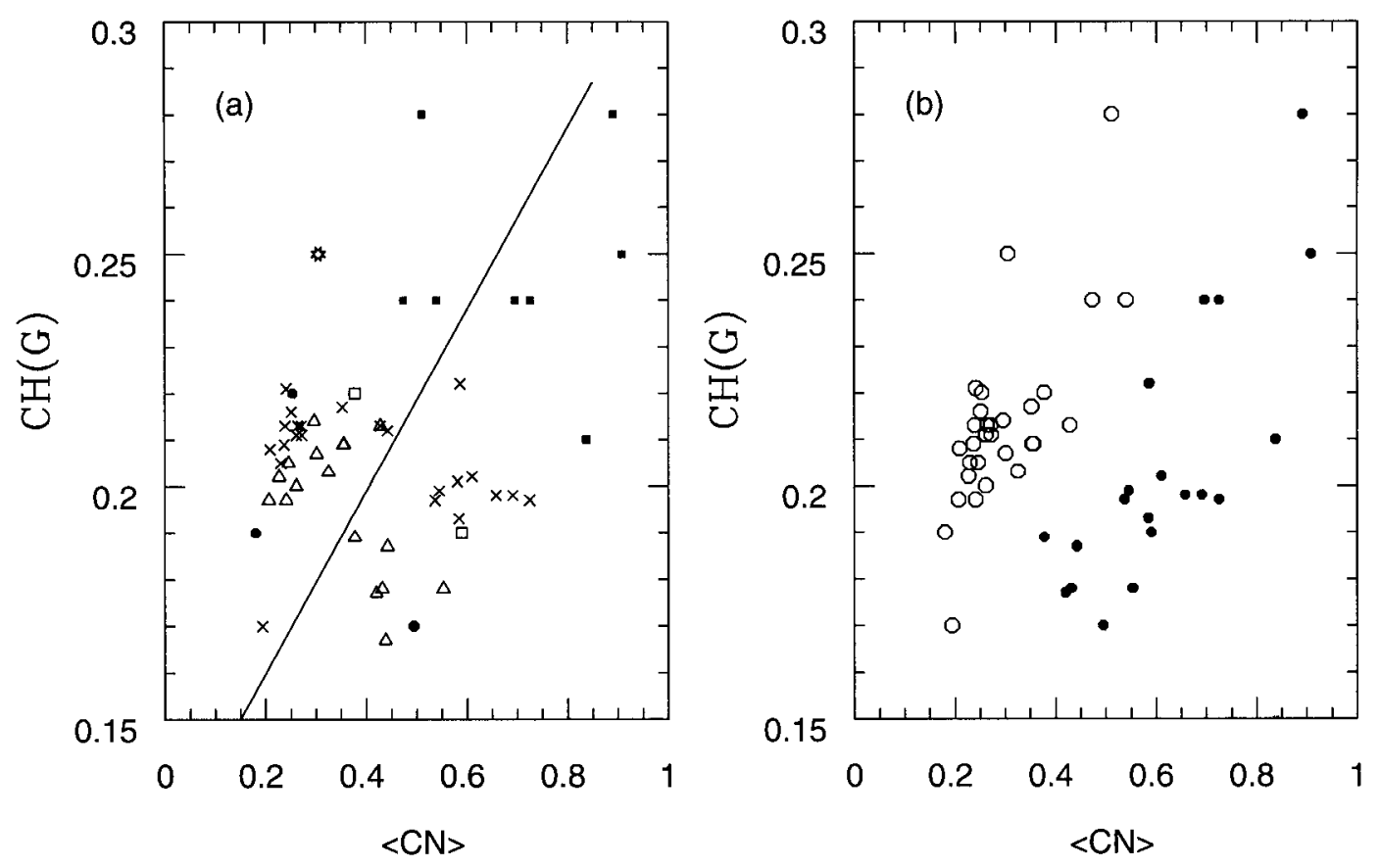

Fig. 4.- a) The $\langle C N\rangle$ index plotted against the $\mathrm{CH}(\mathrm{G})$ index for all stars listed in Table 1. The solid line separated the CN-weak and CN-strong stars. This emprical division of the two classes is based on the study of Cannon et al. (1998). Stars in the lower left are CN-strong, while those in the upper right are CN-weak. They are plotted in Figure 4-b) with symbols of filled circles and open circles respectively. Symbols used in Figure 4-a) are the same as for Fig.1.

But in their Figure 5, no CN-strong star was marked at the position of $\mathrm{I}-111$, which should be at $\mathrm{V}=14.89$ and $m_{C H}=0.290$. However at the position of $\mathrm{V} \sim$ 14.90 and $m_{C H} \sim 0.210$, two CN-strong stars seem to overlap. Therefore it is more likely that the value of $m_{C H}$ for star I-111 in their Table 1 was misprinted. If we assume the value of $m_{C H}$ of the star I-111 is 0.210 , then without exception, there are anti-correlations in the lower RGB and HB.

However for the brighter part of the RGB, the average $\mathrm{CH}(\mathrm{G})$ index of weak-CN stars is 0.243 with a large scatter of 0.024 and that of strong-CN stars is 0.246 with a dispersion of 0.023 . In this part, the index difference between the two groups is too small to conclude any difference, and also it is in the reversed sense of anti-correlation, that is the average $\mathrm{CH}$ index for $\mathrm{CN}$-weak stars is smaller than that for CN-strong stars which is reversed from what is expected from anticorrelation between the two indices. However this could result from the small number of statistics.

Therefore we can conclude that there are two classes, weak-CN stars and strong-CN stars, from the faint RGB all the way to the bright RGB and also in the $\mathrm{HB}$. However the anti-correlation of the $\mathrm{CN}$ and $\mathrm{CH}$ band strengths is found in the $\mathrm{HB}$ and the fainter part of the RGB, with the situation not clear in the brighter part of the RGB.

\section{(b) Spatial Distribution of CN Variations}

Among our sample of 50 stars in M71 which is rather a small sample for statistical purposes, we found 20 $\mathrm{CN}$-strong stars and $30 \mathrm{CN}$-weak stars. And the ratio of CN-strong to CN-weak stars is found to be 0.82 within 2 minutes of arc from the cluster center, while beyond that it decreases to 0.65 . This result is consistent with those of Cohen (1999), and Briley \& Cohen (2001). Cohen (1999) found that there are approximately equal numbers of $\mathrm{CN}$-weak/CH-strong and $\mathrm{CN}$ strong/CH-weak main-sequence stars in M71. Briley \& Cohen (2001) also found remarkably similar numbers of $\mathrm{CN}$-weak and -strong stars and a bimodal distribution of $\mathrm{CN}$ strengths with a pronounced $\mathrm{CN} / \mathrm{CH}$ anti-correlation among M71 giants and main-sequence stars.

It is known that M71 and 47 Tuc have not only a similar metallicity but also similar overall patterns of $\mathrm{CN}$ strengths with a bimodal distribution and a $\mathrm{CN}-$ CH anti-correlation among giants (Briley et al. 2001, Briley 1997, Cannon et al. 1998). However as far as the ratio of $\mathrm{CN}$-strong stars to $\mathrm{CN}$-weak stars is concerned, the two clusters are quite different. In 47 Tuc (Briley 1997) there are much more CN-strong stars than $\mathrm{CN}$ weak stars in RGB and near main-sequence, giving a ratio of 1.8 and the ratio is relatively constant within 10 arc minutes of the cluster center, decreasing to 0.5 at a radius of 16 arc minutes.

Our study also confirms that there are more $\mathrm{CN}$ - 

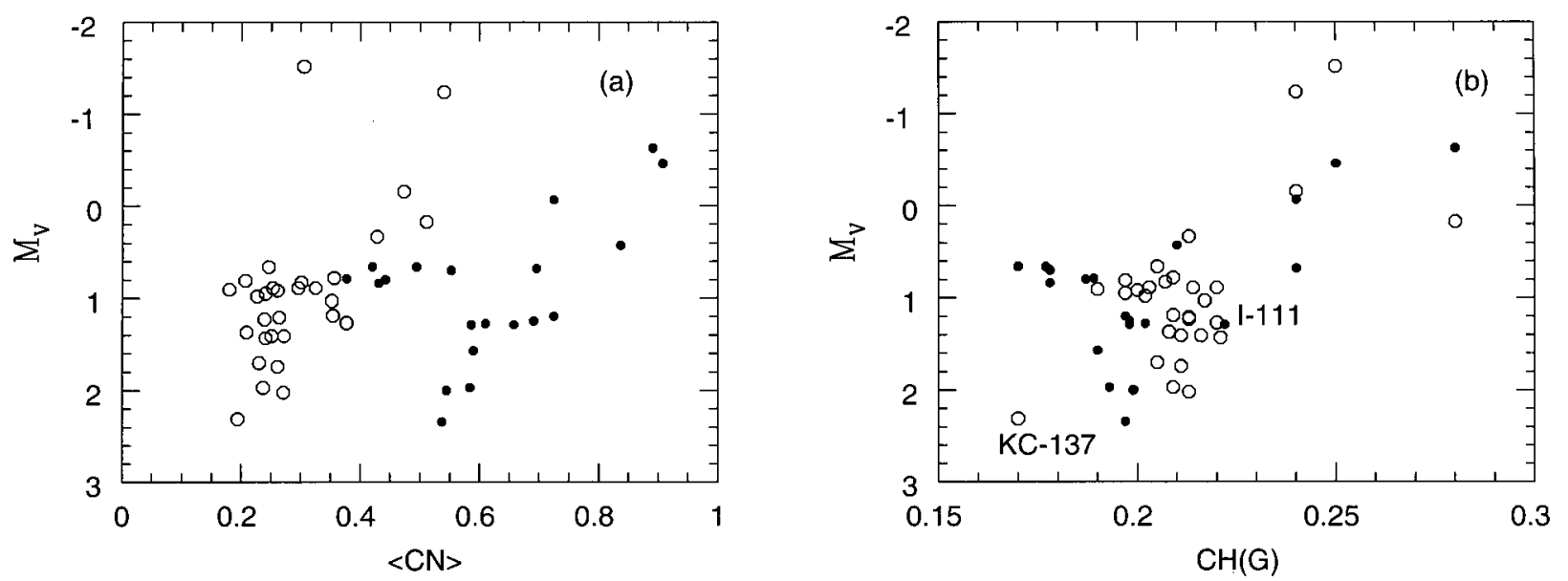

Fig. 5. - Plots of the $\langle C N\rangle$ index and the $\mathrm{CH}(\mathrm{G})$ index against the absolute magnitude for the stars in $\mathrm{M} 71$. The same symbols are used as in Figure 4-b), to denote CN-strong (filled circles) and CN-weak (open circles) stars.

weak stars than CN-strong stars in M71 and the ratio of CN-strong to $\mathrm{CN}$-weak stars decreases to 0.65 in the outer region from 0.82 in the central 2 minutes of arc of M71.

\section{DISCUSSION AND CONCLUSION}

In this study, we have newly obtained $\mathrm{CN}$ and $\mathrm{CH}$ indices for fourteen M71 giants from the archival spectra. Adding published indices for $\mathrm{CN}$ and $\mathrm{CH}$ bands of stars in the M71 cluster, we have reconfirmed that there is a bimodal distribution of CN band strengths among stars from the lower RGB all the way to the upper $\mathrm{RGB}$ and in the HB. However CN-CH anti-correlations are found in lower RGB stars and HB stars, but are not clearly shown among upper RGB stars, which fact may be partly due to small size of the statistical sample, but is worth checking with more sample stars. We have classified CN-weak stars and CN-strong stars using the plot of $\langle C N\rangle$ versus $\mathrm{CH}(\mathrm{G})$ as in Cannon et al. (1998). The ratio of CN-strong to CN-weak stars is 0.82 in the central 2 minutes of arc region and decreases outward to 0.65 . Overall the $r$ value is 0.71 , excluding the variable V2 and a probable non-member KC-137. For each branch of stars, we have found the ratio is 0.69 for the lower RGB, 0.80 for the upper RGB, and 0.60 for the HB. For the upper RGB we exclude the variable star V2. Therefore there seems no evidence that the ratio has been changed according to the evolutionary state.

The observed CN and $\mathrm{CH}$ band strength for $77 \mathrm{M} 71$ main-sequence stars (Cohen 1999) showed also a bimodal distribution with a pronounced $\mathrm{CN} / \mathrm{CH}$ anticorrelation. This pattern was also found in the more luminouse giants by Briley \& Cohen (2001). The ratio I was found to be 0.63 for bright giants and 0.70 for main-sequence stars. Recently Smith (2002) confirmed a correlation between the ratio $r$ of $\mathrm{CN}$-strong to $\mathrm{CN}$ weak stars and the ellipticity $\epsilon$ of clusters, which was found by Norris (1987). It explains that the low value of $\mathrm{r}$ in M71 compared with that in 47 Tuc is because the ellipticity of M71 is zero, while that of 47 Tuc is 0.100 (Norris 1987). However main-sequence stars in 47 Tuc (Da Costa et al. 2004) found a ratio of 0.85 \pm 0.10 which is similar to the ratio of $0.77 \pm 0.11$ far from the center of 47 Tuc (Paltoglou 1989), although the $r$ ratio of the central region of 47 Tuc for the main sequence is 1.8 (Briley 1997). Therefore, the $\mathrm{r}$ ratios only in the central regions of M71 and 47 Tuc are different. This implies that whatever the cause is, the CN enhancement of stars in the clusters are related with their central environments.

\section{ACKNOWLEDGEMENTS}

This work was supported by the SNU foundation \& Overhead Research Fund.

\section{REFERENCES}

Arp, H. C., \& Hartwick, F. D. A., 1971, ApJ, 167, 499

Briley, M. M., 1997, AJ, 114, 1051

Briley, M. M., Bell, R. A., Hoban, S., \& Dickens, R. J., 1990, ApJ, 359, 307

Briley, M. M., \& Cohen, J. G., 2001, AJ, 122, 242

Briley, M. M., Smith, G. H., \& Claver, C. F., 2001, AJ, 122, 2561

Briley, M. M., Smith, V. V., King, J., \& Lamber, D. L., 1997, AJ, 113, 306

Briley, M. M., Smith, V. V., \& Lambert, D. L., 1994, ApJ, 424, L119

Cannon, R. D., Croke, B. F. W., Bell, R. A., Hesser, J. E., \& Stathakis, R. A., 1998, MNRAS, 298, 601

Carbon, D. F., Langer, G. E., Butler, D., Kraft, R. P., Trefzger, C. F., Suntzeff, N. B., Kemper, E., \& Romanishin, W., 1982, ApJS, 49, 207

Cho, Dong-Hwan, \& Lee, Sang-Gak, 2002, AJ, 124, 977 
Cohen, J. G., 1999, AJ, 117, 24

Croswell, K., 1990, Ph.D. Thesis, Harvard Univ.

Cudworth, K. M., 1985, AJ, 90, 65

Da Costa, G. S., Cannon, R., Croke, B., \& Norris, J., 2004, Mem. S. A. It., 75, 370

Freeman, K. C., 1985, in Dynamics of Star Clusters, IAU symposium 113, edited by J. Goodman and P. Hut (Reidel, Dordrecht), 33

Gratton, R. G., Sneden, C., Carretta, E., \& Bragaglia, A., 2000, A \& A, 354, 169

Harbeck, D. Smith, G. H., \& Grebel, E. K., 2003, AJ, 125, 197

Hodder, P. J. C., Nemec, J. M., Richer, H. B., \& Fahlman, G. G., 1992, AJ, 103, 460

Iben, Jr., I., 1964, ApJ, 140, 1631

Iben, Jr., I., \& Renzini, A., 1984, Phy. Reports, 105, 329

Lee, S.-G., 1999, AJ, 118, 925

Lee, S.-G., 2000, JKAS, 33, 137

Norris, J., 1987, ApJ, 313, L65

Norris, J., \& Freeman, K. C., 1979, ApJ, 230, 179

Paltoglou, G., 1989, Ph.D. Thesis, Australian National Univ.

Paltoglou, G., 1990, BAAS, 22, 1289

Penny, A. J., Smith, G. H., \& Churchill, C. W., 1992, MNRAS, 257, 89

Ramirez, S. V., \& Cohen, J. G., 2002, AJ, 123, 3277

Smith, G. H., 2002, PASP, 114, 1215

Smith, G. H., \& Norris, J., 1982, ApJ, 254, 149

Smith, G. H., \& Penny, A. J., 1989, AJ, 97, 1397

Smith, G. H., Shetrone, M. D., Bell, R. A., Churchill, C. W., \& Briley, M. M., 1996, AJ, 112, 1511

Smith, G. H., Shetrone, M. D., Briley, M. M., Churchill, C. W., \& Bell, R. A., 1997, PASP, 109, 236

Sneden, C., Kraft, R. P., Langer, G. E., Prosser, C. F., \& Shetrone, M. D., 1994, AJ, 107, 1773

Suntzeff, N. B., \& Smith, V. V., 1991, ApJ, 381, 160

Sweigart, A. V., \& Mengel, J. G., 1979, ApJ, 229, 624

Trefzger, C. F., Carbon, D. F., Langer, G. E., Suntzeff, N. B., \& Kraft, R. P., 1983, ApJ, 266, 144 